\title{
A bulk-boundary correspondence for dynamical phase transitions in one-dimensional topological insulators and superconductors
}

\author{
N. Sedlmayr, ${ }^{1,2, *}$ P. Jaeger, ${ }^{3,4}$ M. Maiti, ${ }^{5}$ and J. Sirker ${ }^{4}$ \\ ${ }^{1}$ Department of Physics and Medical Engineering, Rzeszów University of Technology, \\ al. Powstańców Warszawy 6, 35-959 Rzeszów, Poland \\ ${ }^{2}$ Department of Physics and Astronomy, Michigan State University, East Lansing, Michigan 48824, USA \\ ${ }^{3}$ Department of Physics, University of Wuppertal, D-42097 Wuppertal, Germany \\ ${ }^{4}$ Department of Physics and Astronomy, University of Manitoba, Winnipeg R3T 2N2, Canada \\ ${ }^{5}$ Bogoliubov Laboratory of Theoretical Physics, Joint Institute for Nuclear Research, 141980 Dubna, Moscow Region, Russia
}

(Dated: December 12, 2017)

\begin{abstract}
We study the Loschmidt echo for quenches in open one-dimensional lattice models with symmetry protected topological phases. For quenches where dynamical quantum phase transitions do occur we find that cusps in the bulk return rate at critical times $t_{c}$ are associated with sudden changes in the boundary contribution. For our main example, the Su-Schrieffer-Heeger model, we show that these sudden changes are related to the periodical appearance of two eigenvalues close to zero in the dynamical Loschmidt matrix. We demonstrate, furthermore, that the structure of the Loschmidt spectrum is linked to the periodic creation of long-range entanglement between the edges of the system.
\end{abstract}

\section{INTRODUCTION}

Two important concepts in modern condensed matter physics are topological and dynamical phase transitions. These two ideas differ from the traditional phenomenology of phase transitions which can be traced back to Landau. ${ }^{1}$ Key to the Landau classification of phase transitions is the concept of order parameters indicative of symmetries broken across phase boundaries. Contrarily, topological phase transitions separate regions with the same symmetries but with different topological properties of the ground state. Dynamical phase transitionsrather than focusing on the non-analyticities which occur in the derivatives of the free energy - concern nonanalyticities which occur in dynamical quantities after a perturbation of the system.

The definition of a dynamical phase transition which we want to follow here is based on the Loschmidt echo ${ }^{2}$

$$
L(t)=\left\langle\Psi_{0}\left|\mathrm{e}^{-i H_{1} t}\right| \Psi_{0}\right\rangle .
$$

Here $\left|\Psi_{0}\right\rangle$ is the initial state of the system before the quench and $H_{1}$ the time-independent Hamiltonian which induces the unitary time evolution of the system. The Loschmidt echo can be viewed as a 'partition function' with fixed boundaries. Similar to the canonical partition function $^{3,4}$ there are 'Fisher zeros' for complex times $t$ with the Loschmidt echo vanishing if the Fisher zeros are real or approach the real axis in the thermodynamic limit. For the Ising model in a transverse field the authors of Ref. 2 have shown that the Loschmidt echo becomes zero at real critical times $t_{c}$ only for quenches across the critical point, i.e. in cases where the initial state is the ground state of the Hamiltonian on one side of the transition while the time-evolving Hamiltonian belongs to the other phase. In this case there is therefore a direct connection between the equilibrium and the dynamical phase transition. In recent years, dynamical phase transitions have also been studied in a number of other models. ${ }^{5-12}$ Contrary to the transverse Ising model it has been found that, in general, there is no connection between equilibrium and dynamical phase transitions: crossing an equilibrium phase transition does not necessarily lead to zeros at real times in the Loschmidt echo while such zeros can also occur for quenches within the same phase. ${ }^{8,13} \mathrm{~A}$ special case are quenches in Gaussian models with topological order. Here it has been shown that, under certain conditions, a quench across a topological phase transition is guaranteed to lead to dynamical phase transitions while the opposite is not true. ${ }^{14}$ The phase of the Loschmidt echo can then be used to define a dynamical topological order parameter which changes at critical times $t_{c}{ }^{15}$

A natural question to ask which we want to address in this manuscript is then: is there a dynamical analogue to the bulk boundary correspondence of equilibrium topological phase transitions? Our paper is organized as follows. In Sec. II we introduce the class of models we will discuss. In Sec. III we review known results for the Loschmidt echo and the return rate in the periodic case. We then present results of numerical calculations of the return rate for open systems in Sec. IV. To understand the origins of the observed sudden changes of the boundary contribution to the return rate at dynamical phase transitions we investigate the dynamical entanglement structure in Sec. V. Our results are summarized in Sec. VI.

\section{MODELS}

We focus here on one-dimensional (1D) models with symmetry protected topological (SPT) phases. Following the ten-fold way symmetry classification ${ }^{16}$ we have three symmetry classes with ground states labeled by a $\mathbb{Z}$ topological invariant AIII, BDI, and CII; and two labeled by a $\mathbb{Z}_{2}$ topological invariant $\mathrm{D}$, and DIII. The 
unitary particle-hole operator $\mathcal{C}$ for BDI, D, and DIII obeys $\{\mathcal{C}, H\}=0$ with $H$ the Hamiltonian of the system and $\mathcal{C}^{2}=1$. The other two symmetries we require are the time reversal symmetries $\mathcal{T}_{ \pm}$satisfying $\mathcal{T}_{ \pm}^{2}= \pm 1$. They must also anticommute with $\mathcal{C}$. BDI has additionally $\left[\mathcal{T}_{+}, H\right]=0$ and a $\mathbb{Z}$ invariant in $1 \mathrm{D}$. DIII has additionally $\left[\mathcal{T}_{-}, H\right]=0$ and a $\mathbb{Z}_{2}$ invariant in $1 \mathrm{D}$. As $\mathcal{T}_{-}$ is the time reversal symmetry of the electrons, DIII has Kramer's pairs. D, with a $\mathbb{Z}_{2}$ invariant in $1 \mathrm{D}$, has no additional symmetry beyond particle-hole. If both $\mathcal{T}_{ \pm}$ symmetries are present the system is best thought of as in BDI with an additional TR symmetry protecting the Kramer's pairs and this will have a $\mathbb{Z}$ invariant in $1 D{ }^{16,17}$

We will use examples principally in the BDI class, which possesses both a unitary 'time reversal symmetry', and a particle hole symmetry. It therefore also possesses chiral or sublattice symmetry. The topological superconductors in which Majorana bound states are sought all belong to either BDI, D, or DIII. ${ }^{18-23}$

For concreteness we consider 1D Hamiltonians, which after a Fourier transform on a periodic lattice, are of the form

$$
H=\sum_{k} \Psi_{k}^{\dagger} \mathcal{H}(k) \Psi_{k} \text { with } \mathcal{H}(k)=\mathbf{d}_{k} \cdot \boldsymbol{\tau}
$$

where $\boldsymbol{\tau}$ is the vector of Pauli matrices acting in some subspace and $\Psi_{k}$ are the appropriate operators for that subspace. This will be particle-hole space for examples such as the Kitaev chain, which is a topological superconductor, or a unit-cell subspace for the Su-SchriefferHeeger (SSH) chain. These two examples will be those we focus on and we will introduce them in more detail below. In general $\mathbf{d}_{k}=\left(d_{k}^{x}, d_{k}^{y}, d_{k}^{z}\right)$, and diagonalizing $\mathbf{d}_{k} \cdot \boldsymbol{\tau}$ one can find $\tilde{\mathbf{d}}_{k} \cdot \tilde{\tau}$ with $\tilde{\mathbf{d}}_{k}=\left(0,0, \epsilon_{k}\right)$ and such Hamiltonians have pairs of eigenenergies $\pm \epsilon_{k}$, a result of the particle-hole symmetry of the Hamiltonians we consider.

\section{A. SSH model}

The first example we will consider is the $\mathrm{SSH}$ model with open or periodic boundary conditions,

$$
H=-J \sum_{j}\left[\left(1+\delta \mathrm{e}^{i \pi j}\right) c_{j}^{\dagger} c_{j+1}+\text { H.c. }\right] .
$$

Here $J$ is the nearest-neighbor hopping amplitude, $\delta$ is the dimerization, and $c_{j}^{\dagger}$ is the creation operator on site $j$. For periodic boundary conditions the sum is taken up to site $N$ and $c_{N+1}=c_{N}$ while the sum is taken up to $N-1$ for open boundary conditions. The main reason to consider this specific model first, is that an exact solution for open boundary conditions exists ${ }^{24,25}$ which depends on a set of parameters determined by non-linear equations. As a result, numerically accurate data for very large open systems can be easily obtained. The system is topologically non-trivial for $\delta>0$. The particle-hole symmetry is then $\psi_{j} \rightarrow \mathrm{i} \psi_{j}^{\dagger}$ and $T_{+}$is $\psi_{j} \rightarrow(-1)^{j} \psi_{j}^{\dagger}$. Note that the phase of $\mathcal{C}$ must be fixed such that $\left\{\mathcal{C}, \mathcal{T}_{+}\right\}=0$.

For periodic boundary conditions the Hamiltonian can be easily diagonalized. Firstly after a Fourier transform and a convenient rotation,

$$
\Psi_{k}^{\dagger}=\sqrt{\frac{2}{N}} \sum_{j=1}^{N / 2} \mathrm{e}^{\mathrm{i} 2 k j}\left(\begin{array}{cc}
1 & 0 \\
0 & \mathrm{e}^{\mathrm{i} 2 k}
\end{array}\right) \underbrace{\left(\begin{array}{c}
c_{2 j-1} \\
c_{2 j}
\end{array}\right)}_{=\Psi_{j}},
$$

the Hamiltonian takes the form (2) with

$$
\mathbf{d}_{k}=(-2 J \cos [k], 2 J \delta \sin [k], 0),
$$

which can be readily diagonalized. The momenta are $k=2 \pi n / N$ with $n=1,2, \ldots N / 2$. The particle-hole symmetry is now $\mathcal{C}=\mathrm{e}^{\mathrm{i} \pi / 2} \boldsymbol{\tau}^{x} \hat{K}$ and $\mathcal{T}_{+}=\hat{K}$, where $\hat{K}$ is the complex conjugation operator.

Although this model has a $\mathbb{Z}$ winding number, the values are nevertheless confined to be either 0 or 1 . Extensions of this model in the same symmetry class but with a higher winding number are however possible. ${ }^{26}$

\section{B. Long-range Kitaev chain}

As our second example we will consider the Kitaev chain of $M$ sites with long-range hopping terms, ${ }^{18}$

$$
\begin{aligned}
H= & \sum_{i, j} \Psi_{i}^{\dagger}\left(\Delta_{|i-j|} \mathrm{i} \tau^{y}-J_{|i-j|} \tau^{z}\right) \Psi_{j+1}+\text { H.c. } \\
& -\mu \sum_{j} \Psi_{j}^{\dagger} \tau^{z} \Psi_{j}
\end{aligned}
$$

with open or periodic boundary conditions. For the periodic case we have $\Psi_{M+1}=\Psi_{1}$. The operators in particlehole space are given by $\Psi_{j}^{\dagger}=\left(c_{j}^{\dagger}, c_{j}\right)$, and $c_{j}^{(\dagger)}$ annihilates (creates) a spinless fermionic particle at a site $j$. In this case we have again $\mathcal{C}=\mathrm{e}^{\mathrm{i} \pi / 2} \boldsymbol{\tau}^{x} \hat{K}, \mathcal{T}_{+}=\hat{K}$, and a Fourier transform brings the Hamiltonian into the form of Eq. (2) where $\Psi_{k}^{\dagger}=\left(c_{k}^{\dagger}, c_{-k}\right)$ and

$$
\mathbf{d}_{k}=\sum_{m=1}^{3}\left(-2 J_{m} \cos [m k]-\mu / 3,2 \Delta_{m} \sin [m k], 0\right)
$$

The long-range hopping has been truncated here at a distance of 3 sites and we define $\vec{J}=\left(J_{1}, J_{2}, J_{3}\right)$ and $\vec{\Delta}=\left(\Delta_{1}, \Delta_{2}, \Delta_{3}\right)$. Note that contrary to the SSH model phases with higher winding numbers in $\mathbb{Z}$ exist, allowing for a more general investigation of quenches between topological phases with different invariants, and therefore also different numbers of boundary states. The momenta are $k=2 \pi n / M$ with $n=1,2, \ldots M$ and the total system size is $N=2 M$. 


\section{THE LOSCHMIDT ECHO AND RETURN RATE}

In this section we will define the quantities studied throughout the following, and review results for periodic boundary conditions. The initial state $\left|\Psi_{0}\right\rangle$ in Eq. (1) is the many-body ground state of the initial Hamiltonian $H_{0}$ before the quench. The unitary time evolution is then determined by the Hamiltonian $H_{1}$. The Loschmidt echo in a translationally invariant system of the form of Eq. (2) can be easily calculated ${ }^{27}$ as the momentum $k$ remains a good quantum number during the quench. One finds

$$
L(t)=\prod_{k}\left[\cos \left(\epsilon_{k}^{1} t\right)+\mathbf{i} \hat{\mathbf{d}}_{k}^{0} \cdot \hat{\mathbf{d}}_{k}^{1} \sin \left(\epsilon_{k}^{1} t\right)\right],
$$

with $\hat{\mathbf{d}}_{k}^{0,1}=\mathbf{d}_{k}^{0,1} / \sqrt{\mathbf{d}_{k}^{0,1} \cdot \mathbf{d}_{k}^{0,1}}$ and $\mathbf{d}_{k}^{0,1}$ being the parameter vector in the Hamiltonian (2) before and after the quench respectively. The product in $k$ is over all filled states of the lower band.

More generally, for any free fermion system the Loschmidt echo can always be obtained from the singleparticle correlation matrix defined by

$$
\mathcal{C}_{i j}=\left\langle\Psi_{0}\left|\Psi_{i}^{\dagger} \Psi_{j}\right| \Psi_{0}\right\rangle .
$$

Here $i$ and $j$ run over all lattice sites. The Loschmidt echo in terms of the correlation matrix is given by ${ }^{28-30}$

$$
L(t)=\operatorname{det} \mathbf{M} \equiv \operatorname{det}\left[1-\mathcal{C}+\mathcal{C} \mathrm{e}^{\mathrm{i} \boldsymbol{H}_{1} t}\right] .
$$

Here $\boldsymbol{H}_{1}$ is the Hamiltonian matrix written in the same basis as $\mathcal{C}$. We will use (10) to calculate $L(t)$ in the open boundary case and call $\mathbf{M}$ the Loschmidt matrix in the following. Eq. (8) is easily recovered for periodic boundary conditions by transforming to momentum space and by using the eigenbasis of the initial (momentum resolved) Hamiltonian.

In a many-body system we expect, in general, an orthogonality catastrophe: the overlap between the initial state and the states in the time evolution will become exponentially small in system size $N$. It is therefore useful to define the return rate as

$$
l(t)=-\frac{1}{N} \ln |L(t)| .
$$

The non-analytic points of the return rate are determined by the zeros of the Loschmidt echo. ${ }^{2}$ Fisher zeros in the complex plane occur in the translationally invariant case at times ${ }^{14}$

$$
t_{n}(k)=\frac{\pi}{\epsilon_{k}^{1}}\left(n+\frac{1}{2}\right)+\frac{i}{\epsilon_{k}^{1}} \tanh ^{-1}\left(\hat{\mathbf{d}}_{k}^{0} \cdot \hat{\mathbf{d}}_{k}^{1}\right)
$$

wit $n$ being an integer. These zeros lie on the real axis and therefore give rise to non-analytic behavior for the return rate at critical times

$$
t_{n}=\frac{\pi}{2 \epsilon_{k^{*}}^{1}}(2 n-1), \text { where } n \in \mathbb{Z},
$$

if a critical momentum $k^{*}$ exists with

$$
\hat{\mathbf{d}}_{k^{*}}^{0} \cdot \hat{\mathbf{d}}_{k^{*}}^{1}=0 .
$$

This is the condition for the vanishing of the imaginary part in Eq. (12). We introduce the critical time scale $t_{c}=\pi /\left(2 \epsilon_{k^{*}}^{1}\right)$. Where multiple critical times exist, we take the smallest critical time to be the timescale $t_{c}$.

\section{BOUNDARY CONTRIBUTIONS TO THE RETURN RATE}

In this study we are interested in the boundary contributions to the return rate (11) for systems with open boundaries. In the large $N$ limit we can expand the return rate as

$$
l(t) \sim l_{0}(t)+\frac{l_{B}(t)}{N} .
$$

Here $l_{0}$ is the bulk contribution which is equivalent to the return rate in the thermodynamic limit for periodic boundary conditions. $l_{B}$ is the boundary contribution which contains information about the topologically protected edge states, as we will demonstrate in the following.

\section{A. Finite-size scaling}

The most straightforward approach to find $l_{B}(t)$ is a numerical calculation of the correlation matrix (9) followed by a finite size scaling analysis of the return rate, Eq. (15). We will discuss the results of such an approach here for both our examples, the SSH and the long-range Kitaev chain.

\section{SSH chain}

We start with the SSH chain where the semi-analytical solution for open boundary conditions allows one to obtain highly accurate results for very large systems. We consider quenches between the topologically ordered phase with edge states $(\delta>0)$ and the topologically trivial phase without edge states $(\delta<0)$ in the halffilled case. If we perform a symmetric quench $\delta \rightarrow-\delta$ then the direction of the quench does not matter for the bulk contribution $l_{0}(t)$ shown in Fig. 1 as is obvious from Eq. (8). In both examples cusps in the bulk return rate are present at the critical times determined by Eqs. (13, 14).

The direction of the quench does, however, strongly affect the boundary contribution $l_{B}(t)$. For a quench from the trivial into the topological phase we find a boundary contribution $l_{B}(t)$ which shows large jumps at the critical times, see Fig. 2. For a quench from the topological into the trivial phase we also observe jumps in $l_{B}(t)$ at $t_{c}$ as 


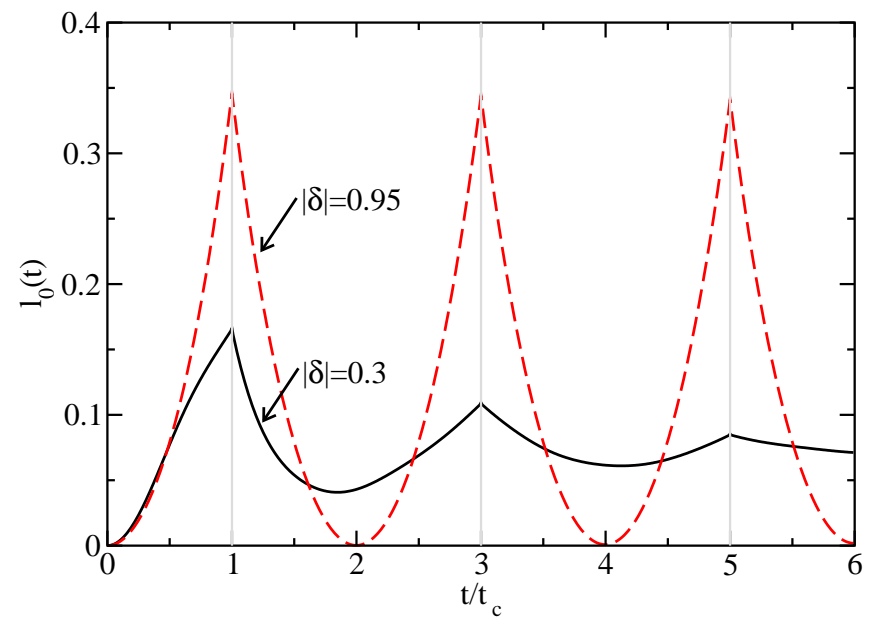

FIG. 1. Bulk contributions for symmetric quenches $\delta \rightarrow-\delta$ in the SSH model for $\delta=0.3$ and $\delta=0.95$.

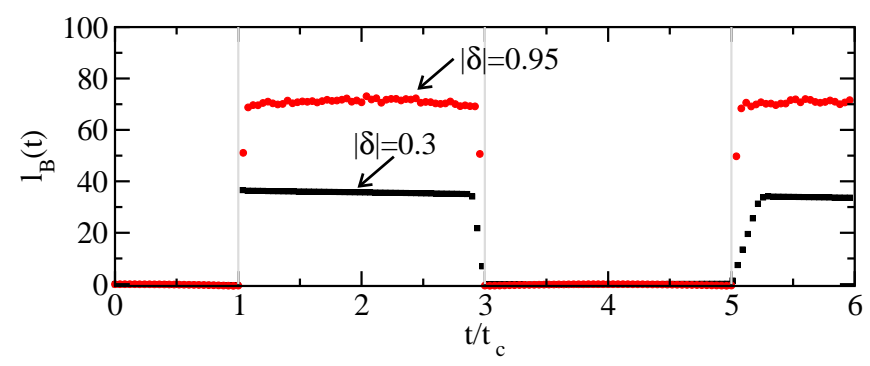

FIG. 2. Boundary contribution to the return rate $l_{B}(t)$ for symmetric quenches from the trivial into the topological phase. $l_{B}(t)$ is extracted from a $1 / N$ scaling analysis of chains of up to $N=2200$ sites.

shown in Fig. 3, however, the jumps are more than two orders of magnitude smaller than for the quench in the opposite direction. The data in the two figures clearly point to a bulk-boundary correspondence: At the same critical times where the bulk contribution shows cusps and the bulk dynamical winding number changes, ${ }^{15}$ the boundary contribution also shows discontinuities. The dependence on the direction of the quench furthermore suggests, that the boundary contribution $l_{B}(t)$ is strongly affected by the presence or absence of symmetry protected edge states in the final Hamiltonian. That the boundary contribution is directly related to the edge states can be seen from the time-dependent occupation of these states, see Fig. 4 . For a system with $N / 2$ spinless fermions in the topological phase, one of the edge modes is filled at time $t=0$ while the other one is empty. At the critical times $t_{c}$, where the cusps in $l_{0}(t)$ occur, both edge modes are approximately half-filled. In the remainder of the paper we will investigate the relation between the edge modes and the singularities in the Loschmidt echo in more detail.

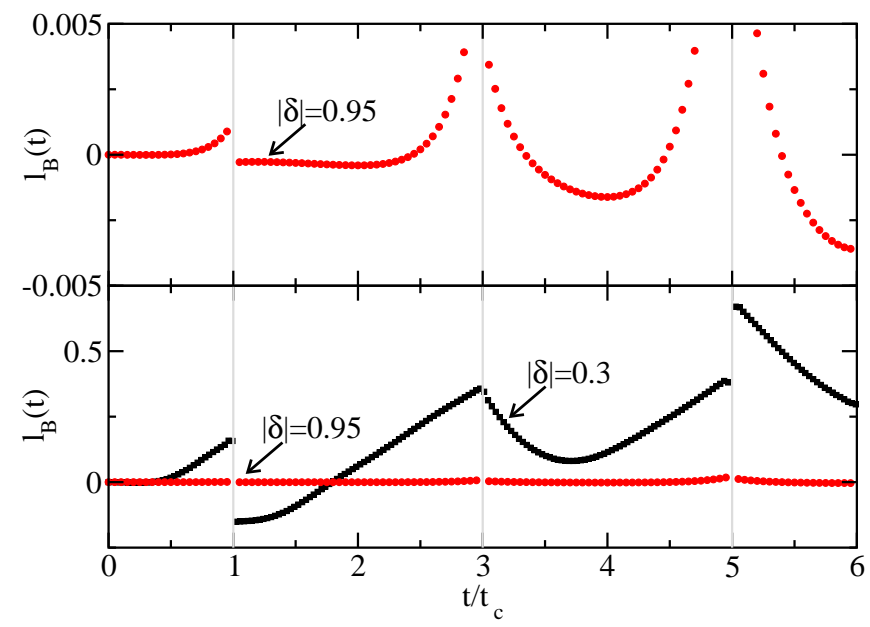

FIG. 3. Boundary contribution to the return rate $l_{B}(t)$ for symmetric quenches from the topological into the trivial phase. $l_{B}(t)$ is extracted from a $1 / N$ scaling analysis of chains of up to $N=2200$ sites. Note the different scale of $l_{B}(t)$ compared to the quenches in Fig. 2. The top panel shows a close-up of $l_{B}(t)$ for the quench from $\delta=0.95$ to $\delta=-0.95$.

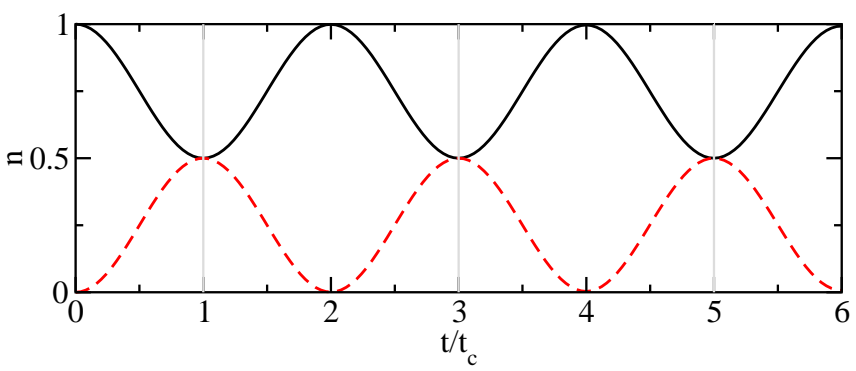

FIG. 4. Occupation of the edge modes for a symmetric quench at $|\delta|=0.95$ from the topological to the trivial phase for a system of size $N=80$.

\section{Long-range Kitaev chain}

Before doing so, we will first present numerical results for $l_{B}(t)$ for the other model system we study here, the long-range Kitaev chain. Contrary to the SSH model, this chain has different topological phases characterized by an integer winding number $\nu_{\mathrm{w}}$. Dynamical phase transitions are expected for any quench between phases with different winding numbers. In Fig. 5 the bulk return rate is shown for two examples. In the case shown in Fig. 5(a) the quench is from $\nu_{\mathrm{w}}=1$ with $\vec{J}=(1,-2,2), \mu=2$, and $\vec{\Delta}=(1.3,-0.6,0.6)$ to $\nu_{\mathrm{w}}=3$ with $\vec{J}=(1,-2,2)$, $\mu=0.1$, and $\vec{\Delta}=(0.45,-0.9,1.35)$. Two critical momenta exist leading to two distinct critical times at which DPT's occur. In Fig. 5(b) a quench from $\nu_{\mathrm{w}}=1$ with $\vec{J}=$ $(1,-2,-2), \mu=3$, and $\vec{\Delta}=(1.3,-0.6,0.6)$ to $\nu_{\mathrm{w}}=-1$ with $\vec{J}=(1,-2,-2), \mu=3$, and $\vec{\Delta}=-(1.3,-0.6,0.6)$ is considered. Cusps in the return rate are again clearly visible, demonstrating that DPT's also occur for quenches 

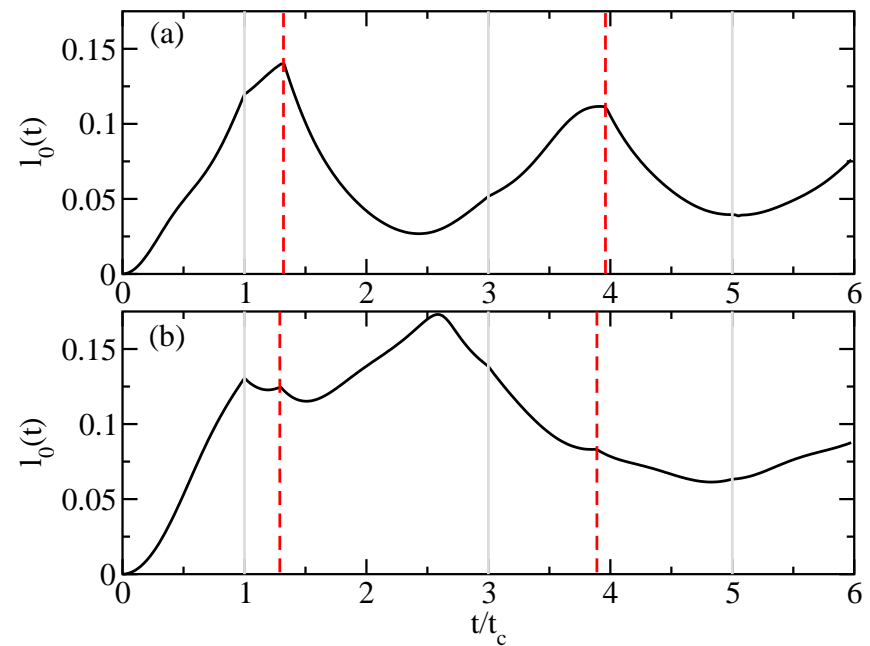

FIG. 5. Bulk return rate for quenches in the long-range Kitaev model from (a) $\nu_{\mathrm{w}}=1$ to $\nu_{\mathrm{w}}=3$ and (b) $\nu_{\mathrm{w}}=1$ to $\nu_{\mathrm{w}}=$ -1 . The gray and dashed red lines show the positions of the critical times.

where only the sign of the winding number changes.

By a finite size scaling analysis of chains up to $N=$ 1600 , we have also extracted the boundary contribution. As an example we show $l_{B}(t)$ for the same quench as in Fig. 5(a) in Fig. 6(a), and for the reverse quench in Fig. 6(b). Note that contrary to the SSH model an analytic solution for the eigensystem is not known so that we cannot increase the system size till a clear scaling also for times very close to $t_{c}$ emerges. Finite-size corrections are still present near DPT's. Furthermore, the edge states have energies which are exponentially close to zero so that an exact diagonalization in multi-precision is required. While the obtained data are therefore not as accurate as for the SSH chain, we nevertheless observe a behavior which is qualitatively similar. At the critical times $t_{c}$ the boundary contribution shows jumps. However, here the jumps are of similar magnitude for both quench directions.

\section{B. Loschmidt eigenvalues}

In order to understand the origin of the discontinuous boundary contribution we next investigate the spectrum of the dynamical Loschmidt matrix $\mathbf{M}$ defined in Eq. (10).

We concentrate first on symmetric quenches in the SSH model for large dimerizations $\delta$ where the structure of the spectrum is particularly simple. In Fig. 7 the spectrum of the matrix $\mathbf{M}$ for a quench from the trivial into the topological phase is shown. At the first critical time $t_{c}$ there are two eigenvalues $\lambda_{1,2}$ whose absolute values become exponentially small in system size. Between $t_{c}$ and $3 t_{c}$ these eigenvalues stay close to zero and this structure repeats itself in time. At $t_{c}$, the argument of the eigenvalues also changes abruptly from zero to $\pm \pi$. These

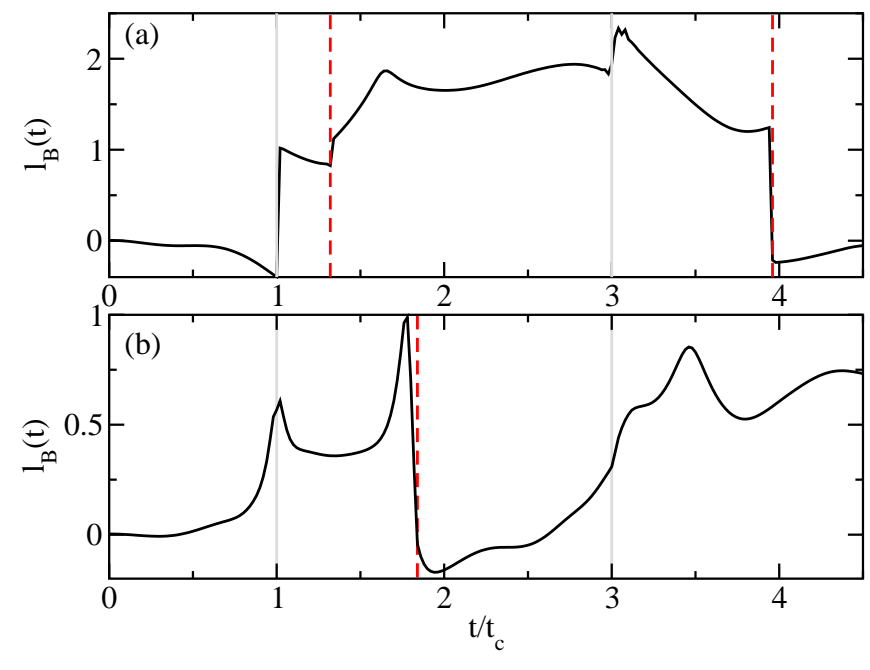

FIG. 6. Boundary contribution $l_{B}(t)$ in the long-range Kitaev model for (a) the same quench as in Fig. 5(a) from $\nu_{\mathrm{w}}=1$ to $\nu_{\mathrm{w}}=3$, and (b) the reverse quench from $\nu_{\mathrm{w}}=3$ to $\nu_{\mathrm{w}}=1$. $l_{B}(t)$ is extracted from a $1 / N$ scaling analysis of chains of up to $N=1600$. The gray and dashed red lines show the positions of the critical times.

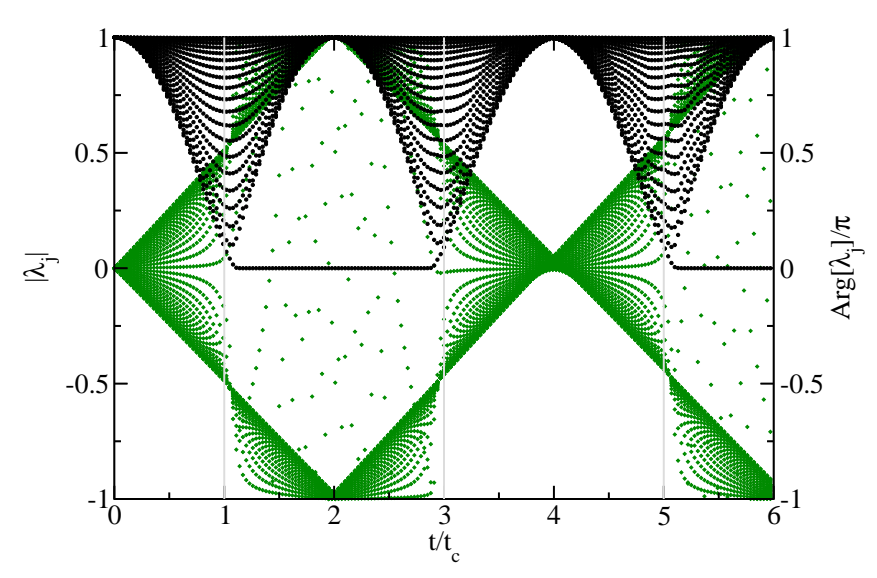

FIG. 7. Absolute value [black circles] and argument [green diamonds] of the eigenvalues of the matrix $\mathbf{M}$ for a symmetric quench at $|\delta|=0.95$ from the trivial into the topological phase for a system of size $N=80$.

two eigenvalues are related to the edges of the system as a comparison to the case with periodic boundary shows where they are absent.

The return rate in terms of the eigenvalues $\lambda_{i}$ of the matrix $\mathbf{M}$ is given by

$$
l(t)=-\frac{1}{N} \sum_{j=1}^{N} \ln \left|\lambda_{j}\right| .
$$

We can isolate the contribution to $l(t)$ from $\lambda_{1,2}$, the two eigenvalues which periodically approach zero:

$$
\Lambda \equiv-\frac{1}{N} \ln \left|\lambda_{1} \lambda_{2}\right|
$$


At a particular system size $N, \Lambda$ reproduces the same behavior as the finite size boundary contribution which we define as $l(t)-l_{0}(t)$, see Fig. 8 . It is the principle source of the large boundary contribution for $t \in\left[t_{c}, 3 t_{c}\right]$.

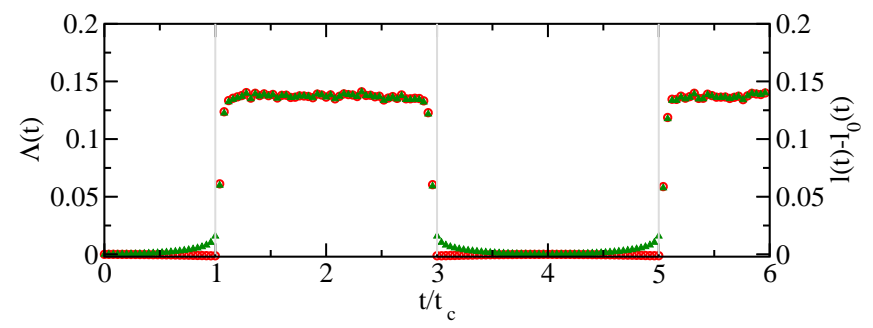

FIG. 8. The boundary contribution to the return rate $l(t)-l_{0}(t)$ [red circles] compared to $\Lambda$ [green triangles], the contribution to the return rate from the two eigenvalues which are close to zero for $t \in\left[t_{c}, 3 t_{c}\right]$, see Fig. 7. Shown for a system of size $N=500$.

At a DPT the system thus not only changes back and forth between the trivial phase with dynamical winding number $\nu=0$ and the topological one with $\nu=1^{15}$ there is also - at the same time - a transition in the edge degrees of freedom. The presence or absence of a pair of zero eigenvalues of the dynamical matrix $\mathbf{M}$ can serve as an equivalent order parameter to the bulk winding number, establishing a concrete bulk-boundary correspondence in this case.

For the quench from the topological to the trivial phase we obtain a slightly different picture. While at the critical times there is still a pair of eigenvalues which approach zero, the eigenvalues no longer remain close to zero for $t \in\left[t_{c}, 3 t_{c}\right]$, see Fig. 9. The direction of the

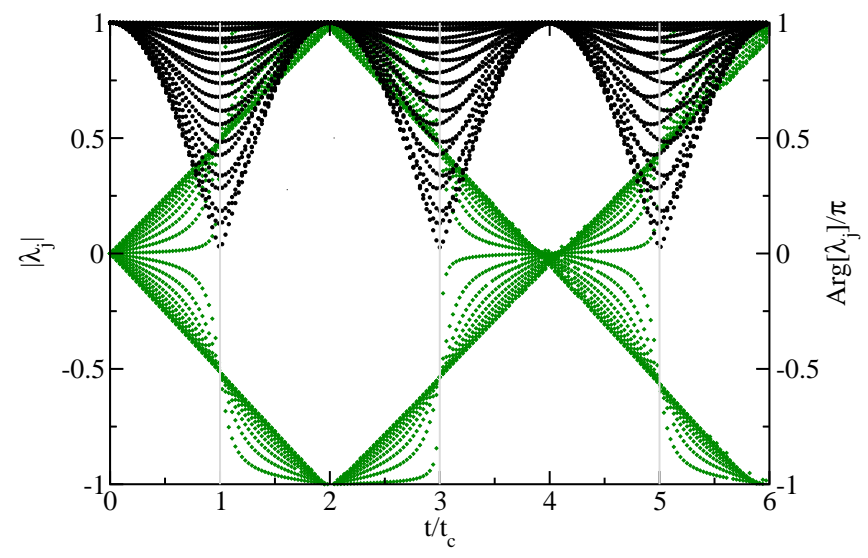

FIG. 9. Absolute value [black circles] and argument [green diamonds] of the eigenvalues of the matrix $\mathbf{M}$ for a symmetric quench at $|\delta|=0.95$ from the topological into the trivial phase for a system of size $N=80$.

quench can thus clearly be distinguished from the spectrum of $\mathbf{M}$. From the almost symmetric spectrum around $t_{c}$ for the topological to trivial quench it is in particular clear that the boundary contribution is of similar magni- tude on both sides of the transition consistent with the scaling results shown in Fig. 3.

So far we have investigated quenches for large dimerizations $|\delta| \lesssim 1$ where the system is almost perfectly dimerized and the Loschmidt spectrum is particularly simple. A natural question to ask is whether or not the spectrum is still useful to detect if edge states are present in the final Hamiltonian for smaller dimerizations. To this end, we present in Fig. 10 data for symmetric quenches at $|\delta|=0.3$. While the spectrum becomes

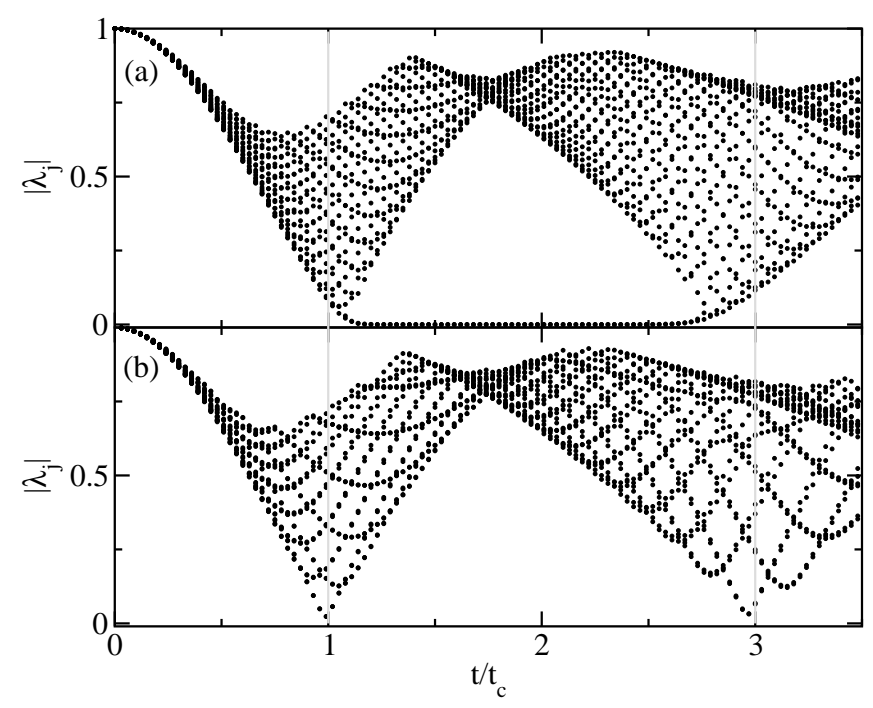

FIG. 10. Absolute value [black circles] of the smallest 20 eigenvalues of the matrix $\mathbf{M}$ for a symmetric quench at $|\delta|=$ 0.3 from (a) the trivial to the topological phase, and (b) vice versa, for a system of size $N=160$.

more complex, the direction of the quench is still obvious. In particular, a pair of eigenvalues close to zero for $t \in\left[t_{c}, 3 t_{c}\right]$ persists for the trivial to topological quench.

In the long range Kitaev model where we are able to consider quenches between more general winding numbers a similar structure is seen in the spectrum of $\mathbf{M}$, see Fig. 11. Although finite size effects are still present, it is already obvious that the results are again very different for the two quench directions. Note that for the quench from $\nu_{\mathrm{w}}=1$ to $\nu_{\mathrm{w}}=3$ the time evolving Hamiltonian, $H_{1}$, has two additional pairs of edge states, compared to the initial Hamiltonian, $H_{0}$. For the quench where the number of edge states increases [Fig. 11(a)] we find, in particular, an extended time interval between critical times where 2 or 4 eigenvalues are zero. In fact two eigenvalues are close to zero for $t \in\left[t_{c}, 3 t_{c}\right]$ and two different eigenvalues are close to zero for $t \in\left[t_{c}^{\prime}, 3 t_{c}^{\prime}\right]$, where $t_{c}^{\prime}$ is the larger of the two critical times for this quench. For the opposite direction, on the other hand, we find a roughly symmetric structure around the first two critical times very similar to the SSH case. Note that finite size effects strongly influence the results for $t / t_{c} \gtrsim 4$. Compatible with the data for the SSH and the Kitaev chain is thus the idea that for the boundary contribution $l_{B}(t)$ 


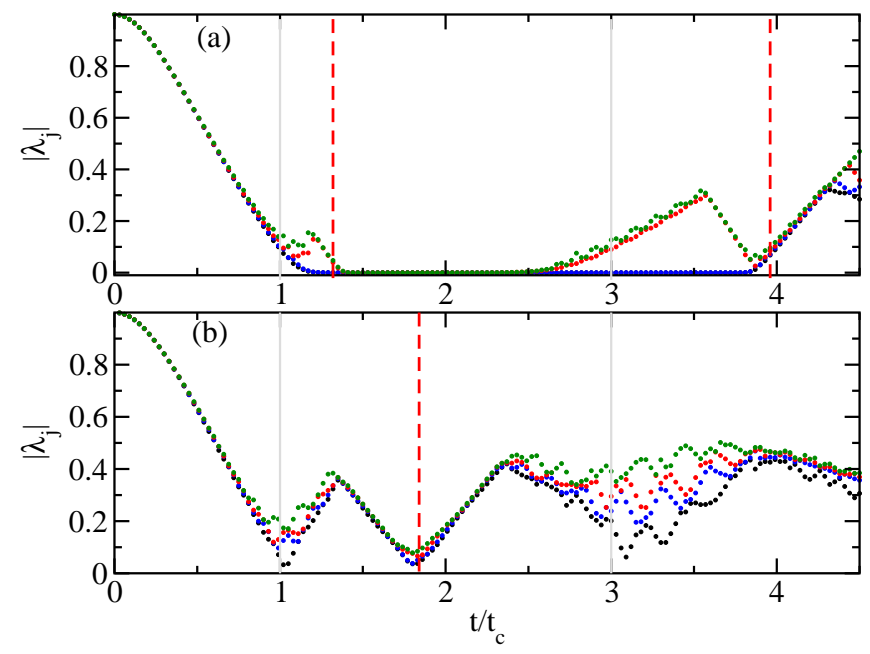

FIG. 11. Absolute value of the smallest four eigenvalues of the matrix $\mathbf{M}$ for the quenches in Fig. 6, for (a) $\nu_{\mathrm{w}}=1$ to $\nu_{\mathrm{w}}=3$, and (b) vice versa, for a system of size $N=600$. Eigenvalues are colored as an aid to the eye.

and for the Loschmidt spectrum, $\operatorname{spec}(\mathbf{M})$, it is important how many more or fewer edge states are present for $H_{1}$ as compared to $H_{0}$.

\section{LONG-RANGE ENTANGLEMENT}

We have established numerically a bulk-boundary correspondence and could show that the spectrum of $\mathbf{M}$ contains information about the edge states. However, the spectrum of this matrix is not an easily measurable quantity and also does not provide a physical picture about what happens to the edge states during the time evolution. In this section we therefore want to connect the changes on the Loschmidt echo with the dynamical entanglement properties of the system. To this end, we consider the entanglement between the two halves of an open chain with an even number of sites. The entanglement entropy is defined as the von-Neumann entropy of a reduced density matrix

$$
S_{\text {ent }}(t)=-\operatorname{Tr}\left\{\rho_{A}(t) \ln \rho_{A}(t)\right\}
$$

with $\rho_{A}(t)=\operatorname{Tr}_{B}|\Psi(t)\rangle\langle\Psi(t)|$ and $|\Psi(t)\rangle=\mathrm{e}^{-i H_{1} t}\left|\Psi_{0}\right\rangle$ being the time-evolved state. The system has been divided up into two blocks $A$ and $B$ of equal size. Here we will focus on the SSH model.

For a Gaussian model the entanglement between a subsystem and the rest can be calculated from the correlation matrix $\mathcal{C}(t)$ defined in Eq. (9) with the now time-dependent two-point correlations restricted to lattice sites within the subsystem. ${ }^{31}$ The entanglement entropy is then given by

$$
S_{\mathrm{ent}}=-\sum_{j}\left[\eta_{j} \ln \eta_{j}+\left(1-\eta_{j}\right) \ln \left(1-\eta_{j}\right)\right]
$$

with $\eta_{j}$ being the eigenvalues of $\mathcal{C}(t)$.

In Fig. 12 the entanglement entropy for a symmetric quench from the trivial into the topological phase at large $|\delta|$ is shown. $S_{\text {ent }}(t)$ is showing oscillations with local

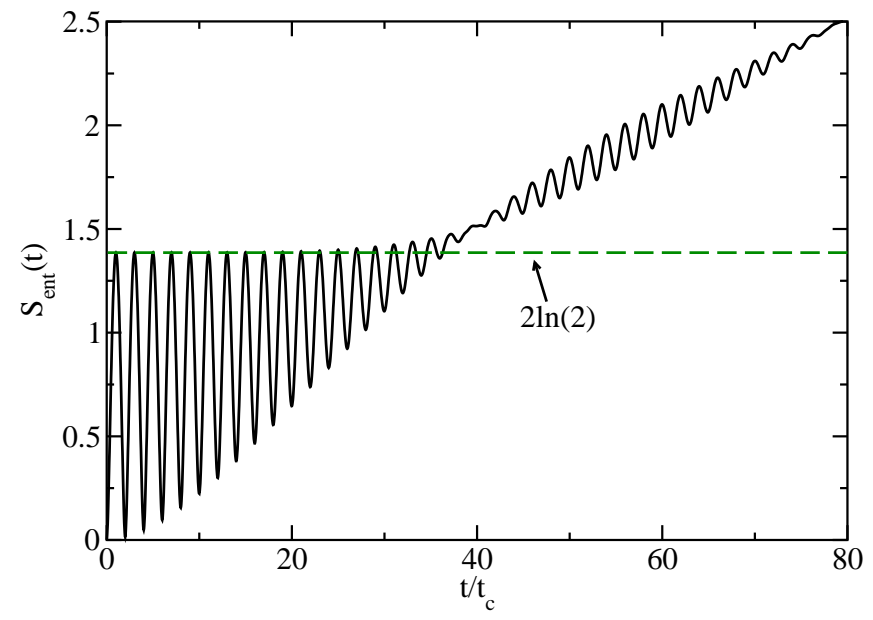

FIG. 12. $S_{\text {ent }}(t)$ between two halves of the system for a symmetric quench at $|\delta|=0.95$ from the trivial to the topological phase with $N=32$.

maxima located exactly at the DPT's. For short times, in particular, $S_{\text {ent }}\left(t_{c}\right) \approx 2 \ln 2$ while at longer times the entanglement entropy on average starts to increase linearly as is expected for a global quench. These observatios can be explained as follows: In the strongly dimerized case, each dimer bond between the two subsystems is in a fully entangled state, $(|10\rangle \pm|01\rangle) / \sqrt{2}$, and contributes $\ln 2$ to the entanglement entropy. The data in Fig. 12 therefore indicate that there are two dimer bonds between the subsystems at the critical times $(2 n+1) t_{c}$. At times $2 n t_{c}$, on the other hand, the two subsystems are almost completely disentangled. This picture is confirmed by directly considering the two-point correlations in the system as a function of time, see Fig. 13. The system

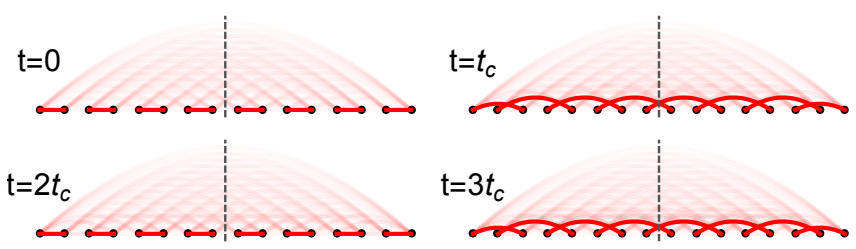

FIG. 13. Time evolution of two-point correlations for the same quench as in Fig. 12 for a system with $N=16$ sites and times $t=0, t_{c}, 2 t_{c}, 3 t_{c}$. The opacity indicates the strength of the correlation and the dashed gray line is the cut between the two subsystems.

starts in a topologically trivial, strongly dimerized state $\left|\Psi_{0}\right\rangle=|\Psi(t=0)\rangle$ and thus $S_{\text {ent }}(t=0) \approx 0$. At the critical time $t_{c}$, two dimer bonds have formed which cross the cut between the subsystems leading to a $S_{\text {ent }}\left(t_{c}\right) \approx 2 \ln 2$. For short times, the system oscillates between these two configurations with other correlations slowly starting to 
build up and finally leading to a linearly increasing entanglement entropy. Note that for a finite system this linear increase will be cut off at $S_{\mathrm{ent}}^{\max }=\frac{N}{2} \ln 2$.

For the quench in the opposite direction, shown in Fig. 14, $S_{\text {ent }}$ also shows oscillations with a frequency set by the critical time $t_{c}$. In this case $S_{\text {ent }}(t=0) \approx 2 \ln 2$

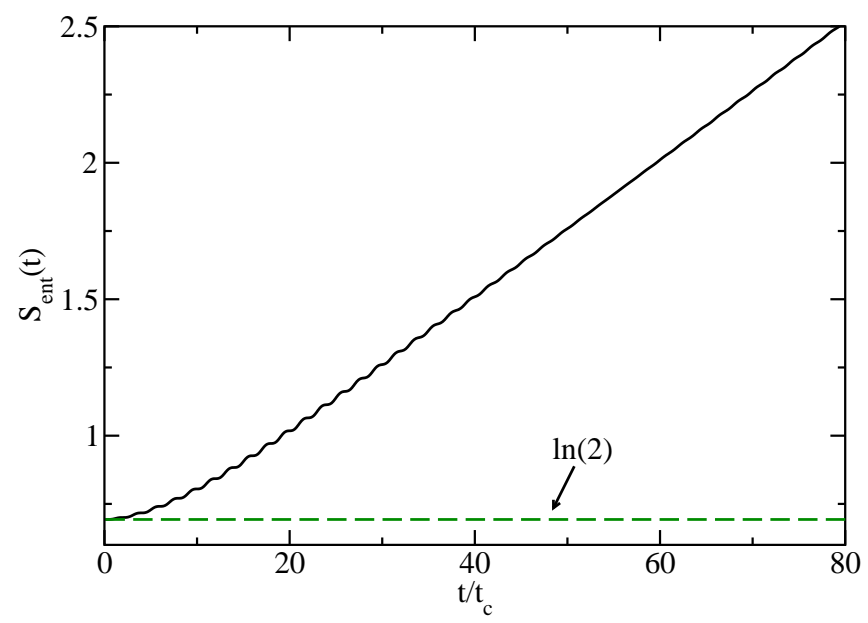

FIG. 14. $S_{\text {ent }}(t)$ between two halves of the system for a symmetric quench at $|\delta|=0.95$ from the topological to the trivial phase with $N=32$.

and for long times the entanglement entropy shows again the expected linear increase. To understand this behavior it is again instructive to consider the time evolution of the two-point correlations, see Fig. 15. At time $t=0$

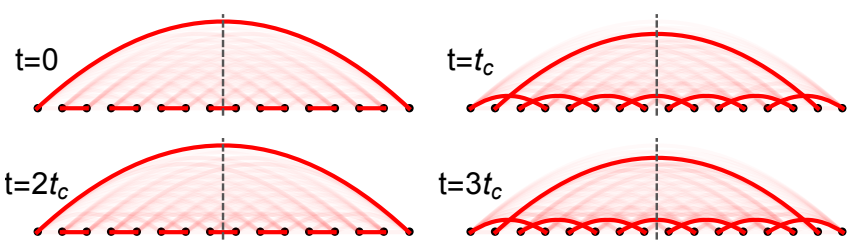

FIG. 15. Time evolution of two-point correlations for the same quench as in Fig. 14 for a system with $N=16$ sites and times $t=0, t_{c}, 2 t_{c}, 3 t_{c}$. The opacity indicates the strength of the correlation and the dashed gray line is the cut between the two subsystems. Long-range entanglement between the edges of the system is present at all times.

a nearest-neighbor dimer and a dimer between the two edge sites is cut. Such long-range entanglement between the boundaries of an open SSH chain has been discussed previously in Ref. 32. Interestingly, the long-range entanglement persists at all times during the unitary time evolution despite the strong quench perturbing the system. While at times $2 n t_{c}$ the two edge sites are strongly entangled, the long-range entanglement moves to the sites one removed from the edge for times $(2 n+1) t_{c}$, see Fig. 15 . The different entanglement structure in the two cases ex- plains the oscillations seen in the entanglement entropy shown in Fig. 14 while the slow build-up of additional correlations explains the linear increase of $S_{\text {ent }}(t)$ at longer times.

\section{CONCLUSIONS}

In this paper we have studied dynamical phase transitions in open chains with symmetry protected topological phases. Specifically, we have concentrated on two examples: the SSH chain and a long-range Kitaev model. In both cases we have shown that for a quench between different topological phases there is not only a cusp in the bulk return rate but also a jump in the boundary $(1 / N)$ contribution. In contrast to the bulk part, the boundary return rate $l_{B}(t)$ is sensitive to the direction of the quench. For the SSH model, in particular, we found that the jump in $l_{B}(t)$ at a DPT is orders of magnitude larger for a quench from the trivial to the topological phase than in the other direction. A clear qualitative difference between the quench directions can also be seen in the Loschmidt eigenvalue spectrum: While for the quench into the topological phase two eigenvalues which behave very differently on both sites of the DPT are largely responsible for the boundary contribution, the spectrum near a DPT is almost symmetric for a quench in the opposite direction.

The critical times $t_{c}$ at which DPT's occur are also clearly visible as oscillations in the entanglement entropy between two halves of the SSH chain. We found that these oscillations can be explained by the time-dependent structure of two-point correlations. At short times and large dimerizations the system oscillates between two configurations of correlations. Starting from the topological phase we discussed, in particular, the long-range entanglement which is transferred from the boundary sites onto the sites one removed from the boundary going from times $2 n t_{c}$ to $(2 n+1) t_{c}$. Quite surprisingly, the longrange entanglement in this case remains stable despite the fact that the quench introduces a large perturbation to the system.

\section{ACKNOWLEDGMENTS}

JS acknowledges support by the Natural Sciences and Engineering Research Council (NSERC, Canada) and by the Deutsche Forschungsgemeinschaft (DFG) via Research Unit FOR 2316. Support for this research at Michigan State University (NS) was provided by the Institute for Mathematical and Theoretical Physics with funding from the office of the Vice President for Research and Graduate Studies. 
* ndsedlmayr@gmail.com

1 L. D. Landau and E. M. Lifshitz, Statistical Physics (Butterworth-Heinemann, Oxford, 1980).

2 M. Heyl, A. Polkovnikov, and S. Kehrein, Phys. Rev. Lett. 110, 135704 (2013).

3 M. E. Fisher, in Lectures in Theoretical Physics. Vol. VIIC - Statistical Physics, Weak Interactions, Field Theory (Univ. Colorado Press, Boulder, 1965).

4 I. Bena, M. Droz, and A. Lipowski, International Journal of Modern Physics B 19, 4269 (2005).

5 A. Mitra, Phys. Rev. Lett. 109, 260601 (2012).

${ }^{6}$ C. Karrasch and D. Schuricht, Phys. Rev. B 87, 195104 (2013).

7 M. Heyl, Phys. Rev. Lett. 113, 205701 (2014).

${ }^{8}$ F. Andraschko and J. Sirker, Phys. Rev. B 89, 125120 (2014).

9 S. Sharma, U. Divakaran, A. Polkovnikov, and A. Dutta, Phys. Rev. B 93, 144306 (2016).

10 C. Karrasch and D. Schuricht, Phys. Rev. B 95, 075143 (2017).

11 A. Gómez-León and P. C. E. Stamp, Phys. Rev. B 95, 054402 (2017).

12 N. Sedlmayr, M. Fleischhauer, and J. Sirker, arXiv: 1711.10381 (2017).

13 S. Vajna and B. Dóra, Phys. Rev. B 89, 161105 (2014).

14 S. Vajna and B. Dóra, Phys. Rev. B 91, 155127 (2015).

15 J. C. Budich and M. Heyl, Phys. Rev. B 93, 085416 (2016).

16 A. P. Schnyder, S. Ryu, A. Furusaki, and A. W. W. Ludwig, Phys. Rev. B 78, 195125 (2008).

17 N. Sedlmayr, M. Guigou, P. Simon, and C. Bena, Journal of Physics: Condensed Matter 27, 455601 (2015).
18 A. Y. Kitaev, Physics-Uspekhi 44, 131 (2001).

19 R. M. Lutchyn, J. D. Sau, and S. Das Sarma, Phys. Rev. Lett. 105, 077001 (2010).

20 Y. Oreg, G. Refael, and F. von Oppen, Phys. Rev. Lett. 105, 177002 (2010).

21 J. Alicea, Y. Oreg, G. Refael, F. von Oppen, and M. P. A. Fisher, Nat Phys 7, 412 (2011).

${ }^{22}$ V. Mourik, K. Zuo, S. M. Frolov, S. R. Plissard, E. P. A. M. Bakkers, and L. P. Kouwenhoven, Science 336, 1003 (2012).

23 C. L. M. Wong and K. T. Law, Phys. Rev. B 86, 184516 (2012).

24 B. C. Shin, Bull. Austral. Math. Soc. 55, 249 (1997).

25 J. Sirker, M. Maiti, N. P. Konstantinidis, and N. Sedlmayr, Journal of Statistical Mechanics: Theory and Experiment 2014, P10032 (2014).

26 M. J. Rice and E. J. Mele, Phys. Rev. Lett. 49, 1455 (1982).

${ }^{27}$ H. T. Quan, Z. Song, X. F. Liu, P. Zanardi, and C. P. Sun, Phys. Rev. Lett. 96, 140604 (2006).

28 L. S. Levitov, H. Lee, and G. B. Lesovik, Journal of Mathematical Physics 37, 4845 (1996).

29 I. Klich, "Quantum noise in mesoscopic physics," (Kluwer Academic Press, Dordrecht, 2003).

30 D. Rossini, T. Calarco, V. Giovannetti, S. Montangero, and R. Fazio, Phys. Rev. A 75, 032333 (2007).

31 I. Peschel, Journal of Physics A: Mathematical and General 36, L205 (2003).

32 L. Campos Venuti, S. M. Giampaolo, F. Illuminati, and P. Zanardi, Phys. Rev. A 76, 052328 (2007). 